\title{
A device for acoustic pulse echo experiments in the SubTerahertz range
}

\author{
R. Legrand, ${ }^{1}$ A. Huynh,,${ }^{1, *}$ S. Vincent, ${ }^{1}$ B. Perrin, ${ }^{1}$ and A. Lemaître ${ }^{2}$ \\ ${ }^{1}$ Sorbonne Universités, CNRS-UPMC Univ. Paris 06, UMR 7588, \\ Institut des Nanosciences de Paris, F-75005 Paris, France \\ ${ }^{2}$ CNRS, UPR 20, Laboratoire de Photonique et de Nanostructures, Route de Nozay, 91460 Marcoussis, France
}

(Dated: January 3, 2017)

\begin{abstract}
We present experimental results on the light-to-sound transduction by a superlattice embedded in an optical microcavity, using picosecond ultrasonics. Experiments on the ability to generate and detect phonons at 0.3 and $0.6 \mathrm{THz}$ were performed at low temperature. Finally, we demonstrate that such a device can detect the acoustic burst that it has generated and which has propagated through the substrate, been reflected on the backside of the substrate and has returned to the device. We study the efficiency of this detection by varying independently the laser wavelength of the pump and probe, by means of asynchronous optical sampling.
\end{abstract}

\section{INTRODUCTION}

Optical microcavities present a unique and versatile way to control light-matter interactions in solids. Many effects have been demonstrated in semiconductor microcavities containing quantum wells, leading to the demonstration of a strong coupling between excitons and photons $^{1}$, parametric oscillations in this regime ${ }^{2}$ or in the weak coupling regime ${ }^{3}$ and Bose-Einstein condensation of polaritons ${ }^{4}$. The modulation of polaritonic modes or a cavity mode is possible with the injection of strain pulses $^{5,6}$ through the microcavity. Conversely, optical microcavities have also been used to control the interaction between light and phonons, leading to the amplification of Raman scattering signal ${ }^{7}$, the enhancement of the coherent generation of high frequency acoustic phonons in an acoustic nanocavity ${ }^{8}$, or obtain high optomechanical coupling rates due to a large overlap between optical and vibrational fields ${ }^{9}$. Superlattices (SLs) are good candidates as phonon generators and are very selective and sensitive detectors too, with the drawback that they emit and detect phonons at different frequencies ${ }^{10}$. Overcoming this problem could be very useful for physical acoustics measurements in the subterahertz range (attenuation, nonlinear acoustics...) and also for the acoustic imaging of embedded nanostructures. SLs are particularly adapted to high frequencies as their period controls the frequency of the phonons which can be generated or detected. By inserting such a structure in an optical microcavity, we take advantage of the amplified photon field and of the modified generation and detection phonon selection rules due to light confinement ${ }^{11}$. Here we present the realization of a coherent monochromatic transducer of high frequency acoustic phonons, acting as a phonon emitter in the underlying substrate, but also capable of detecting the emitted acoustic pulse after a round trip in the substrate. That kind of devices could be very useful to probe or excite nanostructures deposited on the opposite side of the substrate, or to image buried nanostructures.

For that purpose, acoustic wave packets with frequency in the sub- $\mathrm{THz}$ range, with an acoustic wavelength rang- ing from 5 to $500 \mathrm{~nm}$ would be ideal. With SLs, frequency up to the $\mathrm{THz}$ can be obtained in the system GaAs/AlAs with a period as short as $5 \mathrm{~nm}$. Optical to sound transduction in SLs with femtosecond laser pulses is now well established $^{10,12,13}$. The generation is more efficient for phonons with a wave vector $q=0$ while the detection is maximum at modes whose wave vector doubles the light wave vector $q=2 k$. As a result, a phonon generated by a SL is not easily detected by the same SL. This inadequation between generation and detection processes has led to various strategies to optimize both generation and detection, such as using two SLs with a thickness gradient in one of them ${ }^{14,15}$, or inserting the SL into an optical cavity $^{11}$. In the latter case, the efficiency of generation was theoretically analysed inside the cavity. However, the emitted waves from the cavity towards the substrate and its detection were not considered. In this report, the detection of phonons by such a device is studied, using the laser picosecond ultrasonics technique to detect phonons after a round trip in the substrate. Generation and detection are thus decoupled in time. Moreover, to study the effect of the confinement of light separately on both generation and detection, the pump and probe laser beams were independently set at different wavelengths relatively to the microcavity resonance. This was made possible using asynchronous optical sampling (ASOPS) technique.

Experimental setups and samples are described in section II. Section III gives a brief description of the performances of a simple SL deposited on a substrate as phonon emitter and detector. Section IV is devoted to the analysis of a SL embedded in an optical microcavity and Section V provides a summary and conclusions.

\section{EXPERIMENTAL SETUP}

\section{A. Sample description}

The samples were grown by molecular beam epitaxy on (001) oriented $350 \mu \mathrm{m}$ thick wafers of GaAs with both faces highly polished. 
Sample A consists of a simple SL, containing 80 periods of $\mathrm{GaAs} / \mathrm{AlAs}$ bilayers, whose thicknesses are $8.6 / 3.9 \mathrm{~nm}$ so that the period amounts to $d_{S L}=12.5 \mathrm{~nm}$. The periodic modulation of the acoustic impedance, slightly different for GaAs and AlAs, leads to a folding of the acoustic modes into the first Brillouin zone $\left[0 ; \pi / d_{S L}\right]$ and the opening of gaps in the zone center as well as boundaries. The first gap in the Brillouin zone center occurs at $400 \mathrm{GHz}$.

Sample B is a $16.8 \mathrm{~nm}$ period SL embedded in an optical microcavity, as sketched in figure 1(a). The SL has a similar structure as sample A, consisting of 26.5 periods of GaAs/AlAs bilayers. The first gap in the Brillouin zone center is at about $300 \mathrm{GHz}$. The SL itself constitutes the $445 \mathrm{~nm}$ thick optical cavity, forming a $2 \lambda$ cavity $\left(\lambda \approx 760 \mathrm{~nm}\right.$ and $\left.n_{\text {eff }} \approx 3.4\right)$. The reflectors are formed by $51 / 59 \mathrm{~nm}$ AlGaAs/AlAs distributed Bragg reflectors (three pairs for the top mirror and ten pairs for the bottom mirror). Reflectivity measurements reveal a cavity mode at $769 \mathrm{~nm}$ at room temperature and $756 \mathrm{~nm}$ at $15 \mathrm{~K}$ as it can be seen in figure 1(b). These values are well reproduced by the reflectivity calculation based on a matrix transfer method, with the real structure (acoustic SL) inside the Bragg mirrors, taking optical indexes for ambiant or low temperature. At room temperature, the reflectivity measured with the pulsed laser during experiments (red open symbols) shows a slight broadening of the cavity mode, compared to the measurement done with the spectrometer. This may be due to a weak filtering of the laser pulses, as cavity mode and laser pulse widths are comparable. Moreover, the cavity mode width is larger at low temperature as the absorption in $\mathrm{GaAs}$ increases.

It was possible to remove the Bragg reflector by chemical etching to be able to measure the photoluminescence of the sample B SL at $10 \mathrm{~K}$, showing electron-hole transitions at 793 and $800 \mathrm{~nm}$ (E1LH1 and E1HH1 respectively). A maximum of efficiency in optical to sound transduction is expected close to these transitions ${ }^{13}$, so that the SL response as a transducer should be weaker in the wavelength range about the cavity mode, but quasi constant. That offers the advantage of studying only the effects of the optical cavity.

\section{B. Synchronous and asynchronous setup}

First introduced in $1986^{16}$, picosecond ultrasonics has become a standard technique, based on a pump and probe scheme. In such an experiment, a femtosecond light pulse is absorbed by the transducer, here the nanostructure. As a consequence, a strain wave packet is launched, and propagates through the structure then through the substrate. After reflection at the free surface at the backside of the sample, this strain pulse re-enters the nanostructure, changing its optical properties and thicknesses of the different parts of the structure and consequently leads to a change $\Delta R(t)$ in the optical reflec- (a)

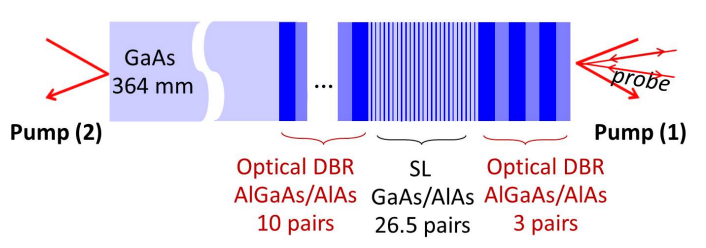

(b)

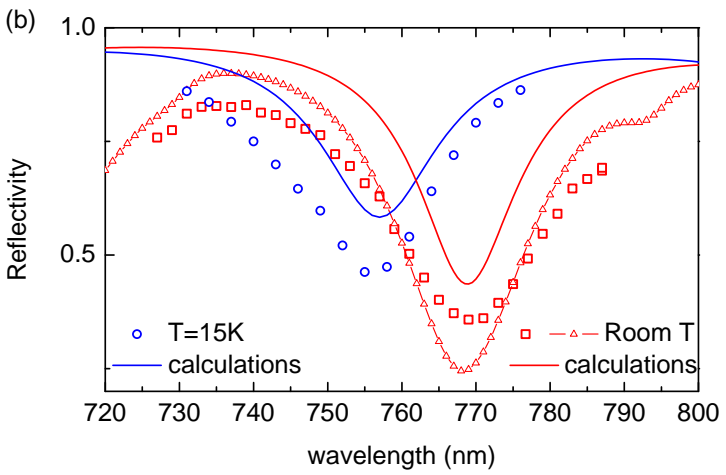

FIG. 1. (a) (color online) Scheme of the sample B, constituted by the acoustic SL inside an optical microcavity, which mirrors are the distributed Bragg reflectors (DBR). The laser beams can be focused on the structure, which is the usual experimental configuration (1), or in acoustic transmission geometry with the pump beam on the back of the substrate (2). (b) Reflectivity of the sample versus light wavelength : measurements at room temperature (red solid line with a spectrometer, red open symbols $\square$ with the femtosecond laser), and at $15 \mathrm{~K}$ (shifted for clarity) (blue open symbols $\circ$ with the femtosecond laser). The calculation done with the real structure inside the cavity is in good agreement (blue solid line).

tivity of the structure. This change $\Delta R(t)$ is measured by a light "probe" pulse that is time-delayed relative to the light pulse used to generate the phonons (the "pump" pulse). The detection setup, previously described ${ }^{17}$, uses a Sagnac interferometer to measure the real and imaginary parts of $\Delta r / r$ where $r$ is the reflection of the electric field. This quantity being of the order of $10^{-5}$ to $10^{-7}$, it is necessary to use lock-in technique or/and signal averaging, with a pump beam modulation of $1 \mathrm{MHz}$. Last, the sample is mounted in a He flow cryostat, in exchange gas, to be cooled down to $15 \mathrm{~K}$ in order to minimize acoustic attenuation during propagation through the wafer. The typical energy of the pump is a few $\mathrm{n} J /$ pulse (except in $\S \mathrm{IV} \mathrm{A}, 9 \mathrm{~nJ} /$ pulse) focused over a $60 \mu \mathrm{m}$ wide spot.

Our setup allows us to focus the pump pulse on the back of the sample (configuration (2) in figure 1(a)). In this case, in acoustic transmission geometry ${ }^{10}$, a broadband pulse is generated and propagates through the substrate to be detected in the same way, by the time-delayed probe focused on the structure.

First, pump and probe pulses are coming from a single femtosecond Ti:Sapphire mode-locked laser providing $80 \mathrm{fs}$ pulses ( $\sim 200 \mathrm{fs}$ pulses incoming on the sample), whose wavelength has been tuned from 720 to $800 \mathrm{~nm}$, with a repetition rate of $\sim 80 \mathrm{MHz}$. The probe pulse is 
time-delayed using a mechanical delay line.

We also used the technique of asynchronous optical sampling (ASOPS), adding a second similar femtosecond laser in the set-up ${ }^{18,19}$. One laser delivers the pump pulses, the other provides the probe pulses with a repetition rate of $f_{p}$ and $f_{s}$ respectively. The repetition rates are slightly different, with an offset of $\delta f \approx 2 \mathrm{kHz}$. As a result, their relative time delay is repetitively ramped from zero to the repetition time $1 / f_{m} \approx 12.5 \mathrm{~ns}$ within the duration $1 / \delta f \approx 500 \mu \mathrm{s}$, making useless the mechanical delay line. The cross correlation of the pump and probe pulses through a BBO crystal triggers the acquisition of the signal which is measured at a cadence of $1 / f_{s}$. We then obtain the signal $\Delta r$ over $12.5 \mathrm{~ns}$ with $\delta f / f_{s}^{2} \approx 0.3 \mathrm{ps}$. Due to the laser jitters, the effective time resolution increases up to $0.5 \mathrm{ps}$ with the number of scans and the pump-probe delay. We paid attention to limit the averaging, and to start the acquisition at the very beginning of the echo or the signal we are interested in and not at the time delay zero, with the help of the mechanical delay line; under these conditions, signals of duration 2 ns were measured with a frequency content up to $350 \mathrm{GHz}$.

\section{PULSE ECHO EXPERIMENT WITH A SL}

\section{A. Selection rules}

In a previous paper ${ }^{13}$, we have studied in detail the detection of an acoustic pulse with a broad frequency spectrum by a SL. The acoustic pulse coming from the substrate and entering in the SL causes a transient change of the optical properties of the SL. First, the refractive index is locally modified due to transient perturbation of the electronic distribution (photoelastic effect proportional to the SL photoelastic parameter $\frac{\partial \epsilon_{S L}}{\partial \eta}{ }_{p h}$ ). Second, the strain due to the acoustic pulse causes a displacement of the surface and the many interfaces (rippling), that leads to an effective photoelastic coefficient $\frac{\partial \epsilon_{S L}}{\partial \eta}$ rip . These effects result in a transient change of the reflectivity of the probe beam, focused on the SL. In an effective medium from the optical point of view, this change is proportional to ${ }^{20}$ :

$$
\begin{array}{ccc}
\frac{\Delta r}{r} \propto & \left({\frac{\partial \epsilon_{S L}}{\partial \eta}}_{p h}+{\frac{\partial \epsilon_{S L}}{\partial \eta} r i p}\right) \\
& \times \int_{0}^{e_{S L}} d z \eta(z) e^{i q z}\left(a_{S L} e^{i k z}+b_{S L} e^{-i k z}\right)^{2}
\end{array}
$$

where $e_{S L}$ is the SL total thickness, $\eta$ the strain, $k$ the effective electromagnetic wave vector in the SL, $a_{S L}$ and $b_{S L}$ the amplitudes of the probe electric field penetrating in the SL and reflected by the interface between the substrate and the SL.

For an optically absorbing SL, $\left|b_{S L}\right| \ll\left|a_{S L}\right|$ and it can be seen that the detection is enhanced for acoustic wave vectors satisfying $q=2 k$ which corresponds to the wellknown back scattering selection rule. When the optical reflection at the interface between the SL and the substrate is not too weak, the cross-product $a_{S L} b_{S L}$ cannot be neglected, then forward scattering phonons $(q=0)$ can also be detected.

Concerning the rippling effect, it can be shown that

$$
\frac{\partial \epsilon_{S L}}{\partial \eta_{\text {rip }}}=-\frac{d_{1} d_{2}\left(C_{1}-C_{2}\right)\left(\epsilon_{1}-\epsilon_{2}\right)}{\left(d_{1}+d_{2}\right)\left(d_{1} C_{2}+d_{2} C_{1}\right)}
$$

where $d_{i}, C_{i}, \epsilon_{i}$ are the thicknesses of the layers, the elastic and electromagnetic dielectric constants for GaAs $(i=1)$ and AlAs $(i=2)$. The rippling contribution amounts to $\frac{\partial \epsilon_{S L}}{\partial \eta}$ rip $=-(0.47+0.007 i)$ which is one order of magnitude lower than the SL photoelastic contribution, dominated by the photoelastic coefficient of GaAs $\left(\left|p_{12}\right| \sim\right.$ a few tens ${ }^{21}$ depending of the wavelength) and can be neglected.

As a SL is able to generate and emit a burst of quasi monochromatic acoustic waves in the substrate, corresponding to the mode $q=0$, these phonons should be detected by this SL.

\section{B. Experimental results}

It is expected in sample A that the SL generates an acoustic wave packet with a spectrum composed of a quasi monochromatic component at $395 \mathrm{GHz}$ corresponding to the $q=0$ mode frequency, superimposed to a low frequency content ${ }^{10}$. As the GaAs substrate is doubleside polished, the emitted acoustic wave can be reflected at the free surfaces at both sides of the sample, while limiting the loss of high frequencies. The experiment consists on the recording of the first echo. One difficulty is that this echo is superimposed to a much larger signal related to photoexcited carriers at the time delay $t=0$ (larger by a factor 500). The tuning of the laser wavelength close to the E1H1 transition $(\sim 780 \mathrm{~nm})$ enhanced the detection of the echo, mainly due to the high values that the photoelastic coefficients can reach around transitions ${ }^{21}$, increasing the detection ${ }^{13}$. This echo is shown in figure 2. Brillouin oscillations from distant echoes are mixed to the signal; the accurate determination of the echo beginning at $t_{0}$ is given by the amplitude jump. Filtering the signal at the $q=0$ mode frequency ( $395 \mathrm{GHz}$ in sample A) reveals a symmetrical signal, corresponding to the back-and-forth in the SL. These results confirm that the SL can generate an acoustic wave packet which can be detected, after propagation, by the SL itself. This is likely due to a good sensitivity with a large number of periods. In the following section, the similar experiment is performed with sample B, with a SL containing a smaller number of periods but embedded in an optical cavity. 


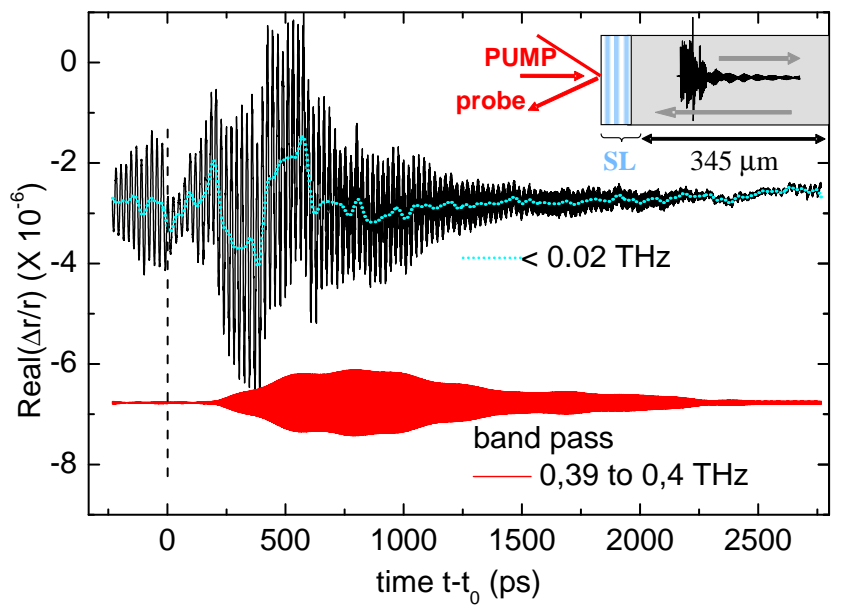

FIG. 2. (color online) In the plain SL (sample A), transient reflectivity corresponding to the first echo as a function of time delay $\left(t_{0} \sim 144 \mathrm{~ns}\right.$ is the time needed for the acoustic burst to make a round trip through the substrate, as sketched in the inset). The dotted curve is a low pass filter $(<20 \mathrm{GHz})$, the red curve is a band pass filter about $400 \mathrm{GHz}$.

\section{STUDY OF THE EFFECT OF THE MICROCAVITY}

By inserting the SL into the optical microcavity as in sample B, we expect to take benefit from a stationary electric field, which implies an efficient detection of the modes $q=0$ and $q=2 k$, but also of a local amplification of the electric field at the cavity mode energy (or wavelength).

\section{A. Experiment: detection}

In this section we explore the detection properties of the device. The experimental configuration "2" is sketched in figure 1(a), where the pump beam is focused on the back of the substrate with a large intensity, typically $9 \mathrm{~nJ} /$ pulse, and absorbed by GaAs on a thickness of about $1 \mu \mathrm{m}$. This leads to the launch of an acoustic pulse with a broad spectral content. Due to nonlinearities during propagation through the thick substrate, the pulse deforms, leading to a spectrum distortion and a generation of high frequencies. As expected, in both samples $\mathrm{A}$ and $\mathrm{B}, q=0$ and $q=2 k$ modes are detected, as it can be seen in figure 3 where the FT transform of typical signals are plotted. The wavelength of the laser (both pump and probe) was tuned to $776 \mathrm{~nm}$ to get the best detection for sample A. However, in sample B, the $q=0$ mode detection is enhanced due to the cavity in comparison with the detection with a single SL (sample A), where it is weakly or not visible: it is the main peak observed in each triplet. It is noticeable that acoustic frequencies up to $1 \mathrm{THz}$ are very well detected, illustrating the high sensitivity of the structure due to the cavity ${ }^{22}$.
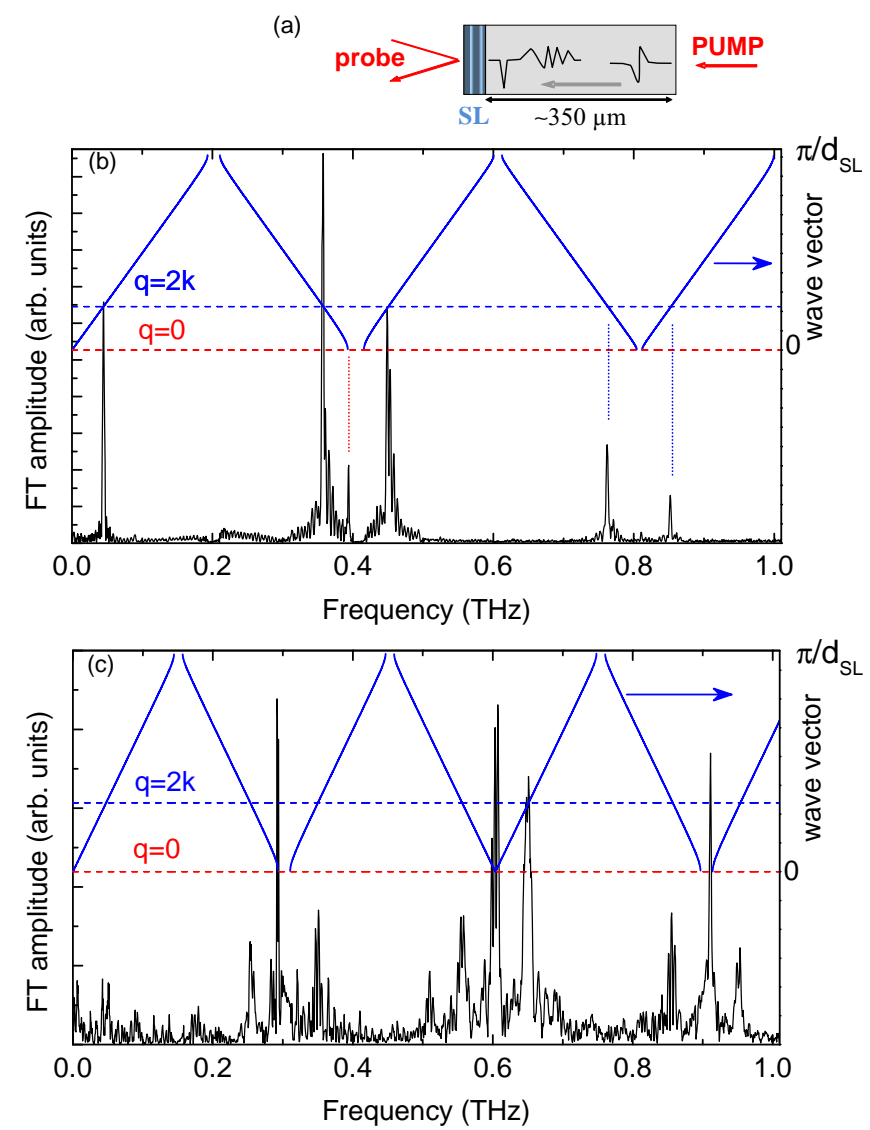

FIG. 3. (color online) FT amplitude of the change of reflectivity, compared to the SL dispersion curve (blue line, right scale), in transmission geometry (configuration (2)) as sketched in (a). (b): in sample A $(776 \mathrm{~nm})(\mathrm{c})$ : in sample B $(754 \mathrm{~nm})$. In sample B, $q=0$ modes detection is enhanced.

\section{B. Temporal signal}

In this last section, the detection of an acoustic burst which had made one round trip through the sample is studied, in the configuration (1) where both pump and probe beams are focused on the device. Just after time delay zero, generation and detection are mixed, as acoustic phonons are detected while the acoustic wave packet builds itself inside the SL and leaks gradually through the substrate (section IVB 1). That is not the case for the echo, for which no study as a function of laser wavelength has been explored (section IV B 2 to section IV B 4).

\section{Before propagation}

Figure 4(a) shows the Fourier transform (FT) amplitude of the signal just after time delay zero between pump and probe, at $\mathrm{T}=15 \mathrm{~K}$ and at a laser wavelength of $750 \mathrm{~nm}$. The slow decay was previously subtracted. Both modes $q=0$ and $q=2 k$ are detected as it can be seen by comparison with the SL dispersion curve. Varying 
the laser wavelength $\left(\lambda_{\text {pump }}=\lambda_{\text {probe }}\right)$, such spectra were measured and normalized by the pump intensity. The FT amplitudes reached at 250 and $290 \mathrm{GHz}$ corresponding to modes in the SL first gap neighborhood, called below modes amplitudes, are plotted in figure $4(\mathrm{~b})$. We show that the mode amplitudes are large only near the cavity mode wavelength. Moreover local maxima are found at wavelengths close to the ones which coincide with the greatest variation of $R(\lambda)$. Note that the error bars due to repeatability amount to 4 to $8 \%$, which is smaller than the amplitude variation around these maxima. This applies to all FT spectra obtained in this paper. In a previous paper ${ }^{11}$, calculations predicted a more efficient generation process for both modes $q=0$ and $q=2 k$ at the laser energy corresponding to the cavity mode, and a more efficient detection at laser energies corresponding to derivative extrema of the probe reflectivity curve or close to these energies, depending on the value of the GaAs photoelastic constant. Indeed, the cavity mode position is modulated by the index or thickness layers changes due to the acoustic generated strain. This variation induces stronger changes in the reflectivity $R_{\text {cav }}(\lambda)$ in spectral regions where the cavity presents the strongest variation in reflectivity (inflection points). Similar results were obtained in a sample containing an acoustic cavity instead of a SL embedded in an optical cavity in ref $^{23}$ figure 3.

\section{First echo after propagation}

We performed a first experiment with a lock-in detection and a modulation frequency at $f_{\text {mod }}=1 \mathrm{MHz}$. The $\mathrm{X}$ and $\mathrm{Y}$ lock-in amplifiers channels recorded on $5 \mathrm{~ns}$ are reported in Figure 5(a). Just after time delay zero, the signal is dominated by carrier excitation, and decays slowly. Let us estimate when the first echo should appear. Let $T$ be the interval between femtosecond laser pulses, and $t_{1}$ the round trip time through the substrate for an acoustic wave. The propagation time being larger than $T$, the echo is detected with a probe pulse coming from $n$ laser pulses after the pump pulse that creates the echo. Thus the echo is expected to occur at a time delay $\Delta t_{1}=t_{1}-n T<T$. Here $t_{1}=152.7 \mathrm{~ns}$, $T=12.44 \mathrm{~ns}$, thus $n=12$ and $\Delta t_{1}=3.38 \mathrm{~ns}$. Indeed, one can observe oscillations at that delay : their amplitude reaches a maximum of $0.05 \times 10^{-3}$ at a laser wavelength of $746 \mathrm{~nm}$ as shown in the inset of figure 5(a) which is 200 times smaller than the electronic background at time delay zero. The $q=0$ mode frequency is present in this feature, as it can be seen in the filtered signal shown in the inset. The lock-in phase was set in order to have the acoustic echo almost entirely on the $\mathrm{X}$ channel, which explains that the large initial signal is split into both channels.

The figure 5(b) shows successive spectra, plotted as a function of time delay, and calculated with a windowed Fourier transform over 500 ps intervals of the signal, centered at regularly spaced time delays. Initially, the rapid
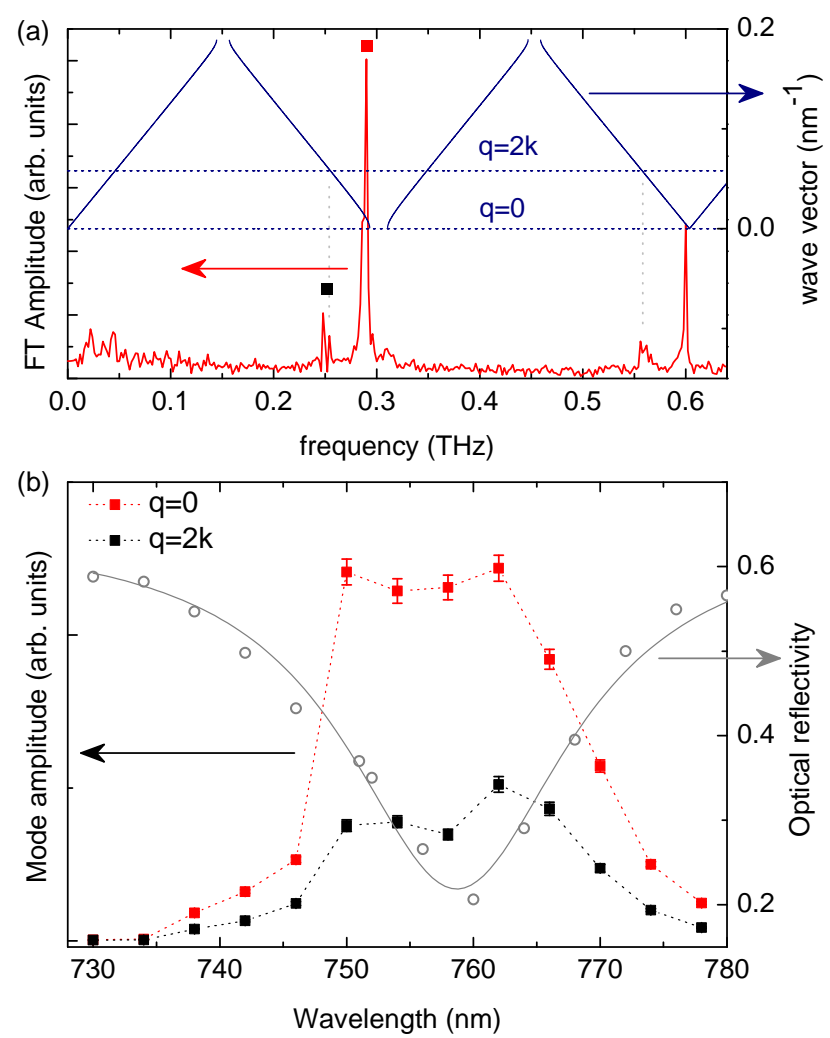

FIG. 4. (color online) In sample B, (a) FT amplitude of the change of reflectivity just after time delay zero (red line), compared to the SL dispersion curve (blue line, right scale). The laser wavelength is set to $750 \mathrm{~nm}$; (b) $q=0$ and $q=2 k$ mode amplitude at 250 and $290 \mathrm{GHz}$ as a function of the laser wavelength. The reflectivity curve (right scale) is reminded (experimental points (o) and calculation (solid curve)).

increase of $\Delta r / r$ due to electronic excitation generates a low frequency continuum, decreasing quickly. All modes are excited too (up to $600 \mathrm{GHz}$ ) and decrease over $2 \mathrm{~ns}$. After a time delay of $3 \mathrm{~ns}$, the detected frequencies correspond mainly to the Brillouin component and $q=0$ mode at $292 \mathrm{GHz}$, in contrast with what is observed at low frequencies just after time delay zero. We can also underline that the echo amplitude only reaches $70 \%$ of the initial amplitude for the $q=0$ mode, measured after time delay zero. As the substrate is not perfectly flat and is epi ready on both faces, this can be attributed to losses during acoustic reflection at the interface between the substrate and the air. The substrate wedge has been estimated to be at most $6 \times 10^{-5}$. Moreover, acoustic attenuation is negligible at that temperature $(\sim 15 \mathrm{~K})$ and that frequency ${ }^{24}$.

It can be seen in figure 5(a) that, for both on $\mathrm{X}$ and $\mathrm{Y}$ channels, acoustic features are superimposed on an electronic background which is larger by two order of magnitude. In order to get rid of this undesired signal in the temporal range where the acoustic echo occurs in one channel, we set the pump modulation frequency at $f_{\text {mod }}=1.637 \mathrm{MHz}$ so that the dephasing between events 

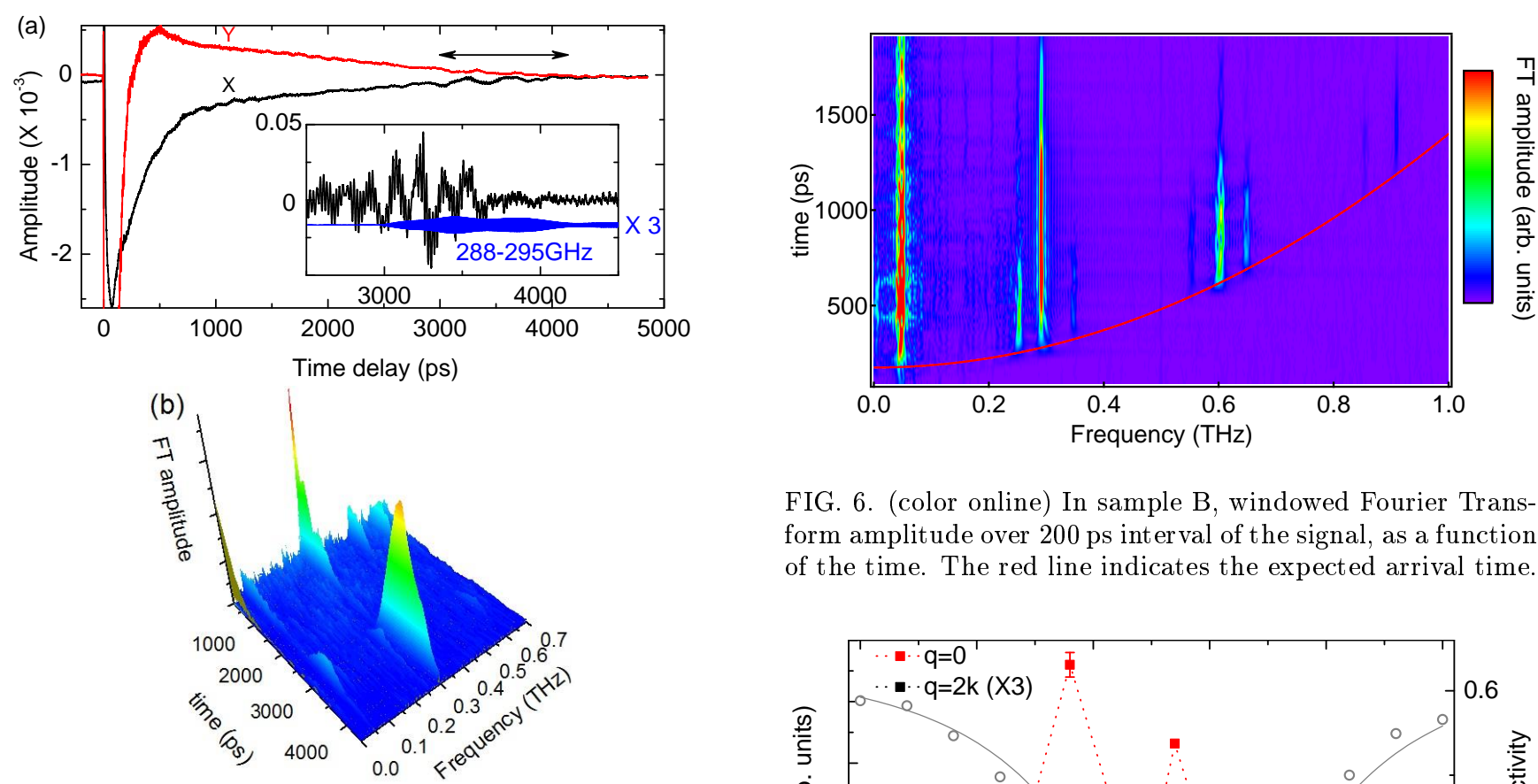

FIG. 5. (color online) In sample B, (a) Transient reflectivity $\operatorname{Re}(\Delta r / r)$ measured by the $\mathrm{X}$ and $\mathrm{Y}$ lock-in amplifier components (the $\mathrm{X}$ component is in phase with the first echo detected about a time delay of $3500 \mathrm{ps}$, after a propagation time of $152.7 \mathrm{~ns}$ ). The laser wavelength is $758 \mathrm{~nm}$ (cavity mode). The arrow indicates the first echo. Inset: zoom of the echo at another laser wavelength $(746 \mathrm{~nm})$ with a band pass filter between 288 and $295 \mathrm{GHz}$ corresponding to $q=0$ mode frequency. (b) Windowed Fourier Transform amplitude over $500 \mathrm{ps}$ interval of the signal, as a function of the time delay. The mode $q=0$ at $293 \mathrm{GHz}$ dominates the $q=2 k$ modes (Brillouin at $48 \mathrm{GHz}$ and $253 \mathrm{GHz}$ ), at the beginning but also in the first echo appearing after $3 \mathrm{~ns}$.

close to time delay zero and the echo is $90^{\circ}$.

We concentrate now on the first echo only. A temporal analysis by windowed Fourier transform over 200 ps intervals, is done for a laser wavelength $\lambda_{\text {pump }}=\lambda_{\text {probe }}=$ $746 \mathrm{~nm}$, where the system response is particularly high, as it can be seen in figure 6 . In addition to Brillouin mode, $q=2 k$ modes are mainly detected in the first two gaps (about 300 and $600 \mathrm{GHz}$ ), weakly in the third one $(900 \mathrm{GHz}) . q=0$ modes are much more efficiently detected as previously noted in $\S$ IV A. Due to dispersion, modes at higher frequencies are detected later, with a delay in agreement with what is expected at the lowest order ${ }^{25} T_{1} \times 12 \gamma \pi^{2} f^{2} / v^{3}$ with $\gamma=0.0074$ and $v=4.78 \mathrm{~nm} / \mathrm{ps}$; This is the red curve plotted in figure 6.

A Fourier analysis of the echo is performed when the laser wavelength varies from 738 to $772 \mathrm{~nm}$ with constant intensity. This leads to a complete different result compared to what happens at time delay zero. A strong amplitude of the backscattering and forward scattering modes is found near the inflection point at low wave-

FIG. 6. (color online) In sample B, windowed Fourier Transform amplitude over $200 \mathrm{ps}$ interval of the signal, as a function of the time. The red line indicates the expected arrival time.

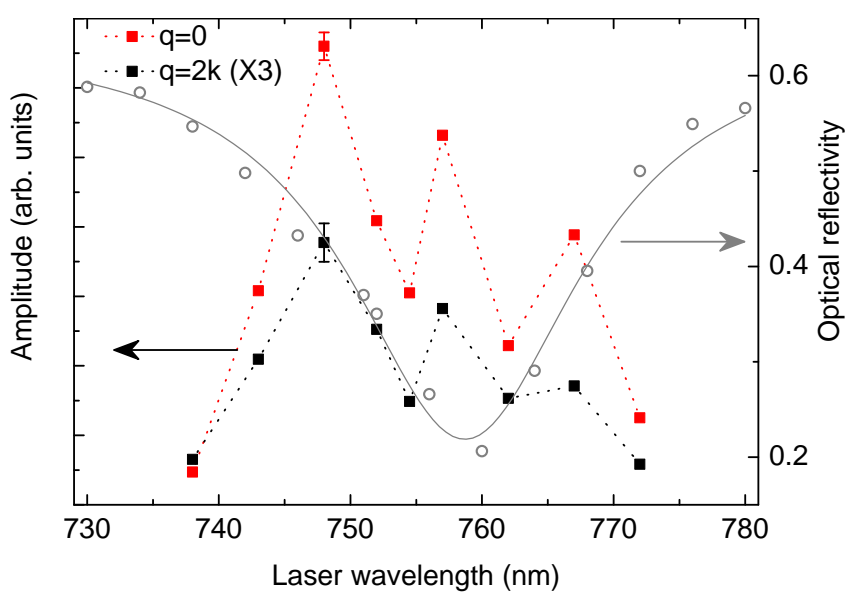

FIG. 7. (color online) In sample B, $q=0$ and $q=2 k$ mode amplitude at 253 and $290 \mathrm{GHz}$ as a function of the laser wavelength (the laser intensity was set to $13 \mathrm{~mW}$ ). The reflectivity curve (right scale) is reminded (experimental points (o) and calculation (solid curve)).

length of the reflectivity curve, as well as two maxima near the cavity mode and at the second inflection point (see figure 7). We will see that this reflects mainly the probe influence.

It is now interesting to determine the experimental conditions to get the best amplitude of the echo. The cavity spectral response has an influence on the detection and generation efficiency. To decouple this influence on both mechanisms, one way is to change the incidence angle of one of the beams, since the cavity spectral response is shifted with angle ${ }^{23}$. Here pump and probe beams are set at almost normal incidence to the sample surface and the wavelength of one of the beam is varied. This was made possible using the ASOPS technique. We then measured the first echo on a duration of $2300 \mathrm{ps}$. By analysing it in the spectral domain, we could study both modes $q=0$ and $q=2 k$ in the first gap $(291 \mathrm{GHz}$ and about $253 \mathrm{GHz})$. 


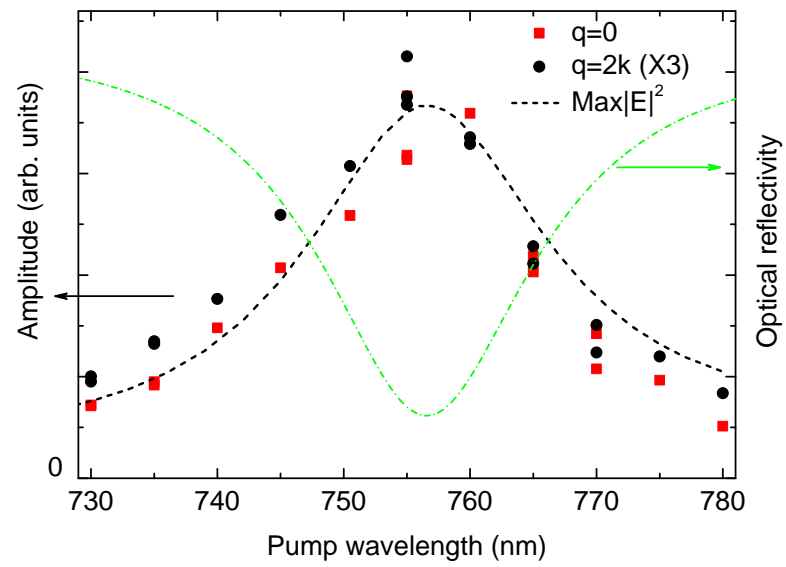

FIG. 8. (color online) In sample B, FT amplitude of the change of reflectivity due to the echo (real part) for $q=0$ at $293 \mathrm{GHz}$ and $q=2 k$ lower mode $(\times 3)$, as a function of the pump laser wavelength. $\lambda_{\text {probe }}=744 \mathrm{~nm}$. In dotted line, electric field square modulus calculated inside the cavity (arb. units), and in dotted dashed line, the corresponding calculated reflectivity (right scale).

\section{Influence of the pump wavelength}

To study the modification on the echo detection due to the pump wavelength, the probe beam wavelength is set to $744 \mathrm{~nm}$ at $1.7 \mathrm{~mW}$ and the pump beam wavelength is varied from 730 to $780 \mathrm{~nm}$, at $44 \mathrm{~mW}$. We checked that this pump intensity does not generate nonlinearity during propagation. The continuous voltage given by the detector, proportional to the optical reflectivity, is recorded simultaneously. FT amplitudes of $q=0$ and $q=2 k$ mode deduced from FT of $\operatorname{Re}(\Delta r)$ due to the first echo are reported in figure 8 . Both modes are maximum when the pump beam wavelength corresponds to the cavity mode. This is expected, as generation mechanisms are proportional to the square modulus of the electric field, which presents its maximum amplification at that wavelength inside the cavity ${ }^{22,26}$. Indeed, the same formalism used to determine the reflectivity of the device in $\S$ II A, calculates the maximum reached by the electric field inside the cavity, and its square modulus is plotted as a function of the electric field wavelength in figure 8. A good agreement is obtained between its variation and that of the modes amplitudes. The parameters used here were: $3.70+0.1 i, 3.68$ and 2.99 for GaAs, GaAlAs and AlAs indexes, 51.3, $59.5 \mathrm{~nm}$ for GaAlAs and AlAs thicknesses of the Bragg mirror, 12, $4.8 \mathrm{~nm}$ for GaAs and AlAs thicknesses in the SL.

\section{Influence of the probe wavelength}

The reverse experiment is conducted: the pump beam is set at a wavelength of $756.7 \mathrm{~nm}$ corresponding to the cavity mode, with an intensity of $44 \mathrm{~mW}$. The genera-

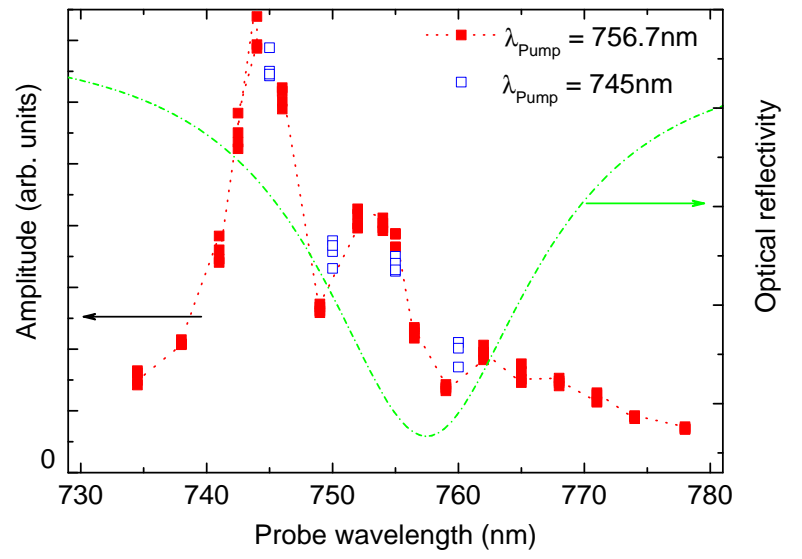

FIG. 9. (color online) In sample B, FT amplitude of the change of reflectivity due to the echo (real part) for $q=0$ at $293 \mathrm{GHz}$ as a function of the probe laser wavelength. The pump beam was set at a wavelength $\lambda_{\text {pump }}=756.7 \mathrm{~nm}$ (red square) or $\lambda_{\text {pump }}=745 \mathrm{~nm}$ (open blue square, $\times 1.8$ ). In dotted dashed line, the calculated reflectivity (right scale).

tion is then maximised, and $\operatorname{Re}(\Delta r)$ is measured as the probe beam wavelength is varied, keeping its intensity set at $1.3 \mathrm{~mW}$. FT amplitudes corresponding to the $q=0$ mode are reported on figure 9 . Three maxima appear, two of them are close to inflection points of the reflectivity curve. The maximum in between is closed to the cavity mode, shifted to low wavelengths. Surprisingly, the mode $q=0$ is overall enhanced near the inflection point at low wavelength. We checked these features by choosing another pump wavelength. At $\lambda_{\text {pump }}=745 \mathrm{~nm}$ and at $\lambda_{\text {probe }}=744 \mathrm{~nm}$, from figure 8 , amplitudes should be smaller by a factor $\approx 1.8$. Taking into account this correction, the results are remarkably consistent, as the measurements with this pump wavelength multiplied by 1.8 match the previous ones (see the blue open symbols in figure 9). The $q=2 k$ mode amplitude follows this variation.

As previously said, one expects that the cavity enhances the detection modes where the reflectivity varies the most (maxima of its derivative, i.e. inflection points), but as already noticed ${ }^{11}$, this intuitive image works for the simple case where the photoelastic constant of GaAs is real. Indeed, when this parameter is complex, there can be two or three maxima whose position is shifted relatively to cavity mode and inflection points. We calculated the complex change of reflectivity of the structure assuming a wave packet incident from the substrate side, with a narrow spectral bandwidth centered on the $q=0$ mode, as it was generated by the SL. Both acoustic and optical fields for a given acoustic frequency and light wavelength were calculated in each layer of the structure by matrix transfer formalism. We then deduce the change of reflectivity in the frequency domain, and consequently the amplitude of the FT of $\operatorname{Re}(\Delta r)$. For GaAs, $p_{12}$ is assumed to be complex, with a modulus equals to a few tens, while for AlGaAs and AlAs, we took negligible val- 
ues. We then had to select the frequency corresponding to the $q=0$ mode in the calculated detection spectrum. Doing so, by adjusting the real and imaginary part of $p_{12}$, we were not able to reproduce the three maxima with their relative importance. In particular, we never obtain a larger peak at low probe wavelength, or two large peaks at wavelengths lower that the cavity mode one.

Moreover, we measured also the imaginary part of $\Delta r$ using the interferometric detection, and the $q=0$ mode amplitude is plotted as a function of the probe wavelength in figure 10(a). We obtain two maxima at wavelengths lower than the cavity mode wavelength. The previous simple model reproduces quite well the relative heights of these two peaks and the gap between them but the entire curve is shifted so that the cavity mode is in between (see Fig. 10(b)). The $q=0$ mode amplitude measured from the real part of reflectivity is reminded on the same figure 10(a): the feature in agreement with the calculation plotted in Fig. 10(b) is the shift between the two curves, although imperfect (see at $744 \mathrm{~nm}$ a maximum of the amplitude of $\operatorname{Re}(\Delta r)$ and the minimum of amplitude of $\operatorname{Im}(\Delta r)$ ). Indeed, the correspondence between the maximum of the real part of reflectivity with a minimum of the imaginary part and vice versa comes from the variation of the cavity reflectivity around the cavity mode: the imaginary part has a N-shape variation while the real part presents a peak.

Due to their derivative nature, photoelastic constants can strongly vary and become important at the vicinity of an electronic transition ${ }^{21,27}$. Assuming negligible values for AlGaAs and AlAs photoelastic constants is correct, since the gaps are well above the laser energy. Concerning GaAs, the energy domain we explore here is just above the first excitonic transitions ( 790 and $800 \mathrm{~nm}$ ), and the GaAs photoelastic constant can vary a lot. In Ref. ${ }^{21}$, multiple GaAs/AlAs quantum wells were studied, containing GaAs wells with a thickness of $17.1 \mathrm{~nm}$. The modulus of $p_{12}=\frac{\partial \varepsilon}{\partial \eta}$ in GaAs was deduced near this transition from Brillouin scattering experiments performed from room temperature to $30 \mathrm{~K}$, and can reach nearly $10^{5}$ at low temperature, at resonancy. Here when the probe wavelength was varied, its energy varies from 60 $\mathrm{meV}$ to $120 \mathrm{meV}$ lower than the energy transition. A refinement of the model is needed, taking into account a variation of $p_{12}$ and indexes, even if the laser wavelength range is quite far from electronic transition.

\section{Conclusion}

To summarize, these two studies define the optimal experimental conditions for both excitation and detection of the $q=0$ mode at $292 \mathrm{GHz}$ after propagation over $740 \mu \mathrm{m}$ : the pump beam has to correspond to the optical cavity mode, while the probe beam has to be at $745 \mathrm{~nm}$, near the inflection point of the cavity reflectivity at high energy. We would like to emphasize the complete
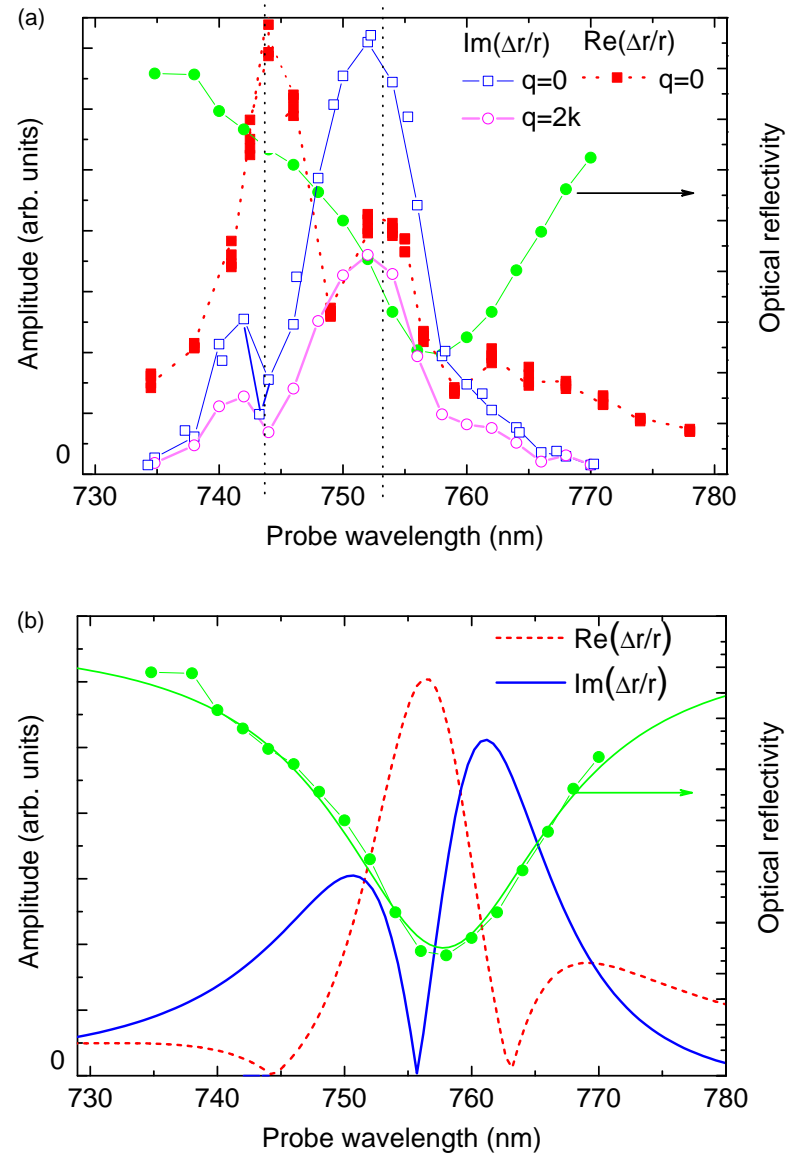

FIG. 10. (color online) In sample B, (a) FT amplitude of the $q=0$ and $q=2 k$ components of the imaginary part of the change of reflectivity due to the echo (open symbols) as a function of the probe laser wavelength. The pump beam was set at a wavelength $\lambda_{\text {pump }}=756.7 \mathrm{~nm}$. Results for the real part $(q=0)$ are rescaled (red square) for comparison; the maxima are indicated by dotted vertical lines. Right scale: measured reflectivity. (b) Results of a simple model for the real and imaginary parts of the FT amplitude of the reflectivity change. Right scale: Reflectivity curve (calculations in solid line, measurements in symbols).

difference between detecting the generated acoustic wave packet after propagation, and detecting the forward and backscattering phonons near time delay zero as it is usually done. Indeed, we refer again to the measurement of the first echo during $3000 \mathrm{ps}$ with the synchronous set-up, with a pump intensity of $13.7 \mathrm{~mW}$ (see figure 7 ). In this experiment where the pump and probe beams have the same wavelength, the mode amplitude versus the laser wavelength varies as the product of the two plots previously obtained, varying only the pump or the probe wavelength. These local maxima, due to probe influence, are not found in the same measurement in the signal just after time delay zero (see figure 4 ).

Moreover we can estimate the gain brought by tuning independently the probe and pump wavelength. Com- 
pared to the optimized situation with a degenerated configuration ( $\lambda=745 \mathrm{~nm}$ ), the double optimization with "two colors" i.e. both generation and detection processes optimization leads to an amplitude of the $q=0$ mode larger by a factor 1.6.

\section{DISCUSSION AND CONCLUSION}

To measure the amplification achieved in the optical cavity, it would be ideal to have a plain SL with the same characteristics. Sample A is a SL with thinner layers (period $\times 0.74)$ that contains 80 periods $(3$ times more than Sample B) and, in addition, for which the laser wavelength could be tuned near the excitonic transition to optimize the detection. Nevertheless, we compare the FT amplitude of the $q=0$ mode calculated on normalized signal obtained with Sample A and B. We got an amplitude of $4-7 \times 10^{-3}$ when $\lambda_{\text {probe }}=\lambda_{\text {pump }}=745 \mathrm{~nm}$ at high pump intensity. Concerning Sample A, as it can be seen in Fig. 2, the amplitude reaches $7.5 \times 10^{-4}$ at a pump intensity of $70 \mathrm{~mW}$. At a similar high intensity of $240 \mathrm{~mW}$, the amplitude would have been amplified by 2.5 (factor previously measured), i.e. $1.9 \times 10^{-3}$. With the optical cavity, the signal at the $q=0$ mode is multiplied by a factor of 2 to 3.7. A better improvement would be obtained with a laser wavelength closer to the excitonic transition and a higher number of periods.

In conclusion, we studied the ability to emit and detect a quasi monochromatic wave packet of phonons, with a device made of a SL embedded in an optical cavity. Using the ASOPS technique, we have found the conditions to optimize the generation and detection processes. The optical resonator amplifies the generation efficiency, but also results in an enhancement of the detection of the $q=0$ mode. This kind of device could likely work up to the terahertz range, which opens the possibility to study elastic, vibrational and adhesion properties of buried nanostructures or nano-objects in an unprecedented frequency range. Another application could be the imaging of buried structures and the control of the selective excitation of nanostructures deposited on the opposite side of the sample, by an acoustic burst with chosen acoustic frequencies.
* Corresponding author: Agnes.Huynh@insp.jussieu.fr

1 C. Weisbuch, M. Nishioka, A. Ishikawa, and Y. Arakawa, Phys. Rev. Lett. 69, 3314 (1992).

2 P. G. Savvidis, J. J. Baumberg, R. M. Stevenson, M. S. Skolnick, D. M. Whittaker, and J. S. Roberts, Phys. Rev. Lett. 84, 1547 (2000).

3 C. Diederichs, J. Tignon, G. Dasbach, C. Ciuti, A. Lemaître, J. Bloch, P. Roussignol, and C. Delalande, Nature 440, 904 (2006).

4 J. Kasprzak, M. Richard, S. Kundermann, a. Baas, P. Jeambrun, J. M. J. Keeling, F. M. Marchetti, M. H. Szymanska, R. André, J. L. Staehli, V. Savona, P. B. Littlewood, B. Deveaud, and L. S. Dang, Nature 443, 409 (2006).

5 A. V. Scherbakov, T. Berstermann, A. V. Akimov, D. R. Yakovlev, G. Beaudoin, D. Bajoni, I. Sagnes, J. Bloch, and M. Bayer, Phys. Rev. B 78, 241302 (2008).

${ }^{6}$ T. Berstermann, C. Brüggemann, M. Bombeck, A. V. Akimov, D. R. Yakovlev, C. Kruse, D. Hommel, and M. Bayer, Phys. Rev. B 81, 085316 (2010).

7 A. Fainstein and B. Jusserand, Phys. Rev. B 57, 2402 (1998).

8 N. D. Lanzillotti-Kimura, A. Fainstein, A. Huynh, B. Perrin, B. Jusserand, A. Miard, and A. Lemaître, Phys. Rev. Lett. 99, 217405 (2007).

9 A. Fainstein, N. D. Lanzillotti-Kimura, B. Jusserand, and B. Perrin, Phys. Rev. Lett. 110, 037403 (2013).

10 A. Huynh, B. Perrin, N. D. Lanzillotti-Kimura, B. Jusserand, A. Fainstein, and A. Lemaitre, Physical Review B 78, 233302 (2008).

11 N. D. Lanzillotti-Kimura, A. Fainstein, B. Perrin, and B. Jusserand, Phys. Rev. B 84, 064307 (2011).

12 M. Trigo, T. A. Eckhause, J. K. Wahlstrand, R. Merlin, M. Reason, and R. S. Goldman, Appl. Phys. Lett. 91,
023115 (2007).

13 A. Huynh, B. Perrin, and A. Lemaître, Ultrasonics 56, 66 (2015).

14 M. F. P. Winter, A. Fainstein, B. Jusserand, B. Perrin, and A. Lemaître, Appl. Phys. Lett. 94, 103103 (2009).

15 A. Huynh, B. Perrin, B. Jusserand, and A. Lemaître, Applied Physics Letters 99, 191908 (2011).

16 C. Thomsen, H. T. Grahn, H. J. Maris, and J. Tauc, Phys. Rev. B 34, 4129 (1986).

17 J.-Y. Duquesne and B. Perrin, Phys. Rev. B 68, 134205 (2003).

18 P. A. Elzinga, R. J. Kneisler, F. E. Lytle, Y. Jiang, G. B. King, and N. M. Laurendeau, Applied optics 26, 4303 (1987).

19 A. Bartels, R. Cerna, C. Kistner, A. Thoma, F. Hudert, C. Janke, and T. Dekorsy, Review of Scientific Instruments 78 (2007), 10.1063/1.2714048.

20 C. Rossignol and B. Perrin, analytical sciences 17, 245 (2001).

21 B. Jusserand, A. N. Poddubny, A. V. Poshakinskiy, A. Fainstein, and A. Lemaitre, Phys. Rev. Lett. 115, 267402 (2015).

${ }^{22}$ Y. Li, Q. Miao, A. V. Nurmikko, and H. J. Maris, J. of Appl. Phys. 105, 083516 (2009).

23 N. D. Lanzillotti-Kimura, A. Fainstein, B. Perrin, B. Jusserand, L. Largeau, O. Mauguin, and A. Lemaitre, Phys. Rev. B 83, 201103 (2011).

24 R. Legrand, A. Huynh, B. Jusserand, B. Perrin, and A. Lemaitre, Phys. Rev. B 93, 184304 (2016).

${ }^{25}$ H.-Y. Hao and H. J. Maris, Phys. Rev. B 63, 224301 (2001).

26 N. D. Lanzillotti-Kimura, A. Fainstein, A. Lemaitre, B. Jusserand, and B. Perrin, Phys. Rev. B 84, 115453 (2011). 
27 P. Etchegoin, J. Kircher, M. Cardona, C. Grein, and E. Bustarret, Physical Review B 46, 15139 (1992). 